\title{
GA AND DERIVATIVE DERIVED GENERALIZED PREDICTIVE CONTROL IN REDUCING BULLWHIP EFFECT
}

\author{
H. W. Gomma, MIEEE \\ Electronics, Communication and Computer Engineering, Department, \\ Helwan University, Egypt \\ (e-mail: $\underline{\text { hgomma@yahoo.co.uk) }}$
}

(Received April 11, 2006 Accepted May 13, 2006)

\begin{abstract}
In supply chains, the phenomenon of bullwhip effect (the variance amplification of order quantities observed in supply chain) has received a considerable attention by companies as it leads to tremendous losses and poor customer services. This variance amplification occurs according to the necessity of using forecasting methods by companies to predict the demand. To overcome this problem the business society resorts to develop what is called decision rules which show that the bullwhip effect is not avoidable. This paper introduces a control engineering approach called Derivative Derived Generalized Predictive Control (DDGPC) together with Genetic Algorithm GA to reduce this effect. The proposed method can reduce bullwhip effect. Moreover, stability and robustness analyses of the proposed technique are investigated. This would help decision-makers in supply chains management to reduce the negative consequences of the bullwhip effect.
\end{abstract}

KEYWORDS: Supply Chains, Bullwhip Effect, GA, Predictive Control

\section{INTRODUCTION}

In 2004 IEEE Conference on Control Applications, the control scientists have called for a new control slogan and named it "Look Around You". The spirit of this slogan is the main motif to introduce this paper. This paper shows to the business people that using control engineering in supply chain management could have a significant impact in solving complicated management problems. One of the noticed problems by the business society, is that fast changes in demand by customers normally leads to a great loss either by slow response to this demand which leads to product shortage or by extra production that causes unwanted inventory stocks. This problem has firstly been analyzed in $[1,2]$ through the so called supply chains. It was found that the increase of orders occurs as one moving up the supply chain. Later, $P \& G$ called this phenomenon the bullwhip effect while in some industries it is known as the "whiplash" or the "whipsaw" effect [3]. Lee et al [3] attributed the bullwhip effect to the five major causes: order batching, price fluctuation, rationing and shortage gaming processing, the use of demand forecast updating and non-zero lead times. The adjusting of demand forecast and as a result adjusting the parameters of the inventory replenishment rule has shown over reactions to short term fluctuations can lead to variance amplification 
$[4,5]$. In addition, they have shown that the order up to polices always generate a bullwhip effect, based on the control engineering approach. The analysis was based on a transfer function for a particular order up to policy after using the so called causal loop diagram. A special predictive control technique called Delayed Generalized Predictive Control (DGPC) [6] was introduced to that model. The results showed the strength of the DGPC in reducing the Bullwhip effect [7]. On the other hand, Sterman [8] developed a new replenishment rule based on fractional adjustments which generated smooth order patterns. Although the new rule is considered as a successful mechanism to dampen order fluctuations when decision makers have to rely on forecasts, variance amplification in orders was still observable and recovery from and the time taken for the net stock to recover completely from this step input signal was found to be longer. This indicates extra cost.

This paper proposes two different approaches to reduce the bullwhip effect namely Genetic algorithm (GA) and Derivative Derived Generalized Predictive Control (DDGPC) [9]. GAs are searching algorithms based on simulation of the human trial and -error procedure using the "survival of the fittest" Darwinian principle [10] as they have been successfully applied in many optimization problems [10-12]. Therefore, they can be used to minimize the difference between the orders and the demands (bullwhip effect). On the other hand, the main objective of the DDGPC is to improve the system performance in terms of the system overshoot without adding computational complexity and with the ability to derive an analytical solution to the control problem. Therefore, this concept can be introduced to the supply chain to reduce bullwhip effect in a robust manner in the presence of an inaccurate lead time, production time and forecast estimates. The DDGPC controller incorporates many advantages can be summarized as follows: an optimizer with significant strength in reducing system overshoot and it can be easily tuned to achieve certain degree of stability, performance, and robustness.

The paper is organized as follows. In section 2, the basic DDGPC algorithm is presented. The proposed replenishment rule and its transfer function with stability analysis are given in section 3 . In section 4 , the GA technique is introduced to the system. Then, in section 5, the controller is designed using DDGPC, supported by stability/robustness analyses and simulation examples. Simulation results are presented and discussed in section 6. The paper ends with summary and conclusion in section 7 .

\section{THE OPTIMAL PREDICTION FOR DERIVATIVE DERIVED GENERALIZED PREDICTIVE CONTROL (DDGPC)}

The main design target of the DDGPC [9] is to calculate a sequence of future control signal in such a way that it minimizes a multistage cost function defined over a horizon. This cost function includes a quadratic function measuring the difference between the predicted system output and certain predicted reference sequence over socalled prediction horizon plus a quadratic function measuring the control efforts, plus the derivative of the future output. This can be written as follows

$$
\left.J\left(N_{1}, N_{2}, N_{u}\right)=E\left\{\sum_{j=N_{1}}^{N_{2}} \delta(j)[\hat{y}(t+j \mid t)-n(t+j)]^{2}+\frac{1}{T_{s}}[\hat{y}(t+j+1 t)-\hat{y}(t+j \mid t)]^{2}\right\}+\sum_{i=1}^{N_{u}} \lambda(i)[\Delta u(t+i-1)]^{2}\right\}
$$


where $\hat{y}(t+j \mid t)$ is the $j$-step ahead prediction of the system on data up to time $t$, $w(t+j)$ is the future reference trajectory, $E\{$.$\} denotes the expectation operator and$ has been used to indicate that the control values chosen are calculated conditioned upon the data available up to and including time $t$ and presuming the stochastic disturbance model. $N_{1}, N_{2}$, and $N_{\mathrm{u}}$ are the minimum costing horizon, maximum costing horizon and control horizon, $\delta(j)$ and $\lambda(j)$ are weighting function to penalize the error and the control sequence respectively. $T_{s}$ is the sampling time.

To minimize the above function the future values of the output $\hat{y}(t+j)$ should be obtained using the system model. For this purpose, consider the following single-inputsingle-output (SISO) plant:

$$
A\left(z^{-1}\right) y(t)=B\left(z^{-1}\right) u(t-1)+C\left(z^{-1}\right) \frac{\zeta(t)}{\Delta}
$$

where $y(t)$ is the output, $u(t)$ is the control sequence, $\zeta(t)$ is the zero mean white noise, $\Delta$ is the differencing operator $1-z^{-1}$ and $A, B$ and $C$ are polynomials in the backward shift operator $\left(z^{-1}\right)$.

$$
\begin{aligned}
& A\left(z^{-1}\right)=1+a_{1} z^{-1}+a_{2} z^{-2}+\cdots \cdots+a_{n a} z^{-n a} \\
& B\left(z^{-1}\right)=b_{0}+b_{1} z^{-1}+b_{2} z^{-2}+\cdots \cdots+b_{n b} z^{-n b} \\
& C\left(z^{-1}\right)=1+c_{1} z^{-1}+c_{2} z^{-2}+\cdots \cdots+c_{n c} z^{-n c}
\end{aligned}
$$

for simplicity the $C$ polynomial is chosen to be 1 or $C^{-1}$ is truncated and absorbed into $A$ and $B$ polynomials. The following Diophantine equation can be used to generate the predicted output:

$$
1=E_{j}\left(z^{-1}\right) \tilde{A}\left(z^{-1}\right)+z^{-j} F_{j}\left(z^{-1}\right)
$$

where $\tilde{A}\left(z^{-1}\right)=A\left(z^{-1}\right) \Delta$. For a unique solution the degree of the polynomials $E_{j}$ and $F_{j}$ should be equal to $j-1$ and $n_{a}$ respectively. For simplicity, $N_{1}=1, N_{2}=N_{u}=N, \delta=1$ and $\lambda(j)=\lambda$.

The output predictions could be stated as:

$$
\begin{aligned}
& \hat{y}(t+1 \mid t)=G_{1} \Delta u(t)+F_{1} y(t) \\
& \hat{y}(t+2 \mid t)=G_{2} \Delta u(t+1)+F_{2} y(t) \\
& \quad \vdots \\
& \hat{y}(t+N \mid t)=G_{N} \Delta u(t+N)+F_{N} y(t)
\end{aligned}
$$

which can be written as:

$$
\begin{aligned}
& \mathbf{y}=\mathbf{G u}+\mathbf{F}\left(z^{-1}\right) y(t)+\mathbf{G}^{\prime}\left(z^{-1}\right) \Delta u(t-1) \\
& \mathbf{y}=\mathbf{G u}+\mathbf{f}
\end{aligned}
$$

where $\mathrm{f}$ is the free response and $\mathrm{Gu}$ is the force response where 


$$
\begin{aligned}
& \mathbf{y}=\left[\begin{array}{llll}
\hat{y}(t+1 \mid t) & \hat{y}(t+2 \mid t) & \cdots & \hat{y}(t+N \mid t)
\end{array}\right]^{T} \\
& \mathbf{u}=\left[\begin{array}{llll}
\Delta u(t) & \Delta u(t+1) & \cdots & \Delta u(t+N-1)
\end{array}\right]^{T} \\
& \mathbf{G}=\left[\begin{array}{cccc}
g_{0} & 0 & \cdots & 0 \\
g_{1} & g_{0} & \cdots & 0 \\
\vdots & \vdots & \vdots & \vdots \\
g_{N-1} & g_{N-2} & \cdots & g_{0}
\end{array}\right], \mathbf{F}\left(z^{-1}\right)=\left[\begin{array}{c}
F_{1}\left(z^{-1}\right) \\
F_{2}\left(z^{-1}\right) \\
\vdots \\
F_{N}\left(z^{-1}\right)
\end{array}\right] \\
& \mathbf{G}^{\prime}\left(z^{-1}\right)=\left[\begin{array}{c}
\left(G_{1}\left(z^{-1}\right)-g_{0}\right) z \\
\left(G_{2}\left(z^{-1}\right)-g_{0}-g_{1} z^{-1}\right) z^{2} \\
\cdot \\
\left(G_{N}\left(z^{-1}\right)-g_{0}-g_{1} z^{-1}-\cdots-g_{N-1} z^{-(N-1)}\right) z^{N}
\end{array}\right]
\end{aligned}
$$

Now the cost function in Equation (2) can be written as:

$$
\mathbf{J}=\mathbf{e}^{\mathrm{T}} \mathbf{e}+\mathbf{D}^{\mathrm{T}} \mathbf{D}+\lambda \mathbf{u}^{\mathrm{T}} \mathbf{u}
$$

where

$\mathbf{e}=(\mathbf{G u}+\mathbf{f}-\mathbf{w})$

$\mathbf{D}=\left[\left(\mathbf{G}_{2} \mathbf{u}+\mathbf{f}_{2}\right)-\left(\mathbf{G}_{1} \mathbf{u}+\mathbf{f}_{1}\right)\right]$

and

$\mathbf{G}_{1}$ is $\left(N_{2}-N_{1}\right) \times N_{u}$ submatrix of the matrix $\mathrm{G}$, consisting of its rows with numbers from $N_{1}$ to $N_{2}-1$.

$\mathbf{G}_{2}$ is $\left(N_{2}-N_{1}\right) \times N_{u}$ submatrix of the matrix G, consisting of its rows with numbers from $\left(N_{1}+1\right)$ to $N_{2}$.

$\mathbf{f}_{1}$ is $\left(N_{2}-N_{1}\right) \times 1$ subvector of the vector $\mathrm{f}$, consisting of its elements with numbers from $N_{1}$ to $N_{2}-1$.

$\mathbf{f}_{2}$ is $\left(N_{2}-N_{1}\right) \times 1$ subvector of the vector $\mathrm{f}$, consisting of its elements with numbers from $\left(N_{1}+1\right)$ to $N_{2}$.

$$
\mathbf{w}=\left[\begin{array}{llll}
w(t+1) & w(t+2) & \cdots & w(t+N)
\end{array}\right]^{T}
$$

To minimize the $J$, assuming that there are no constraints in the control signals, is found to be

$$
\mathbf{u}=-\mathbf{H}^{-1}\left[\mathbf{G}_{2}^{\mathrm{T}}\left(\mathbf{f}_{2}-\mathbf{f}_{1}\right)+\mathbf{G}_{1}^{\mathrm{T}}\left(\mathbf{f}_{1}-\mathbf{f}_{2}\right)+\mathbf{G}^{\mathrm{T}}(\mathbf{f}-\mathbf{w})\right]
$$

where

$$
\mathbf{H}=\left(\mathbf{G}_{2}^{\mathrm{T}} \mathbf{G}_{2}-\mathbf{G}_{2}^{\mathrm{T}} \mathbf{G}_{1}-\mathbf{G}_{1}^{\mathrm{T}} \mathbf{G}_{2}+\mathbf{G}_{1}^{\mathrm{T}} \mathbf{G}_{1}+\mathbf{G}^{\mathrm{T}} \mathbf{G}+\mathbf{I}\right)
$$


the first element, $\Delta u(t)$, of the matrix $\mathrm{u}$, will be applied to the system and will be repeated at every sampling period. In non-adaptive design with a time invariant model, this leads to a time invariant controller. In general, to reduce the computation needed in DDGPC, it is assumed that the control signals will be constant after the control horizon. The performance analysis of the above control laws is summarized in the following section.

\section{THE REPLENISHMENT RULE-FOR ORDERING}

In order up to polices, the bullwhip is unavoidable when demand forecasting is necessary [4]. Accordingly, Dejonkheere et al [4] proposed a new general replenishment rule to reduce variance amplification even when demand should be forecasted. The following exponential smoothing forecast method was used to predict the demand

$$
\hat{D}_{t}=\alpha D_{t}+(1-\alpha) \hat{D}_{t-1}
$$

It should be mentioned that the order decision is made at the end of the period, therefore the current demand can be used in the forecast $\hat{D}_{t}$. For the above exponential smoothing the average age of the data in the forecast is equal to $T_{a}=(1-\alpha) / \alpha[4]$. The proposed new rule was able to derive smoother ordering patterns compared to those obtained by the order up to policies. However, it could not eliminate the bullwhip effect. This can be regarded to the selection of the rule parameters and the limited ability to deal with frequently switching demand quantities up an down. Therefore, the right selection of those parameters using GA will be the objective of the next section while in this section a brief review of the so called replenishment rule is given.

The suggested replenishment rule can be described in words as "ordering quantities are set equal to the sum of forecasted demand, a fraction of $\left(1 / T_{n}\right)$ of the discrepancy of finished goods net stock, and a fraction $\left(1 / T_{w}\right)$ of on order position discrepancy [4]. The order quantity in period $t, O_{t}$, is given by

$$
O_{t}=\hat{D}_{t}^{T a}+\frac{1}{T_{n}}\left(T N S_{t}-N S_{t}\right)+\frac{1}{T_{w}}\left(D W I P_{t}-W I P_{t}\right)
$$

where

$O_{t} \quad$ is the ordering decision made at the end of period $t$.

$\hat{D}_{t}^{T a} \quad$ is the demand forecast (using simple exponential smoothing, see Equation (9) with parameter $T_{a}$.

$T N S_{t} \quad$ is a target net stock level,

$N S_{t} \quad$ is the current net stock in period t,

$D W I P$ is the desired WIP level, and

$W I P_{t} \quad$ is the current work in process (or on order) position in period $t . T N S_{\mathrm{t}}$ is the target net stock level, similar to the safety stock in order-up-to policies. It is updated every period according to the new demand forecast and equals $D_{t}^{T a}$.

$D W I P_{t}$ is updated every period as well, $D W I P_{t}=T_{p} \hat{D}_{t}^{T a}$. 
It should be mentioned that we only have $T_{p}$ orders in WIP and $T_{a}, T_{n}$ and $T_{w}$ can be regarded as the key parameters or controllers of the decision rule. The decision rule of Equation (10) and small variations of this rule have been described by [13]. Analyzing this replenishment rule from a control engineering perspective offers powerful insights into the variance amplification issue.

Using the above equations has led to the following transfer function between the demand and the orders

$$
\frac{O(z)}{D(z)}=\frac{z^{T_{p+1}}\left(-\left(1+T_{a}\right) T_{w}+T_{n}\left(T_{p}+T_{w}\right)(-1+z)+\left(2+T_{a}\right) T_{w} z\right)}{\left(T_{a}(-1+z)+z\right)\left(T_{w}+T_{n}\left(-1+\left(1+T_{w}(-1+z)\right) z^{T p}\right)\right)}
$$

This has been achieved by obtaining the z-transform for the exponential smoothing algorithm

$$
F(z)=\frac{\hat{D}(z)}{D(z)}=\frac{\alpha}{1-(1-\alpha) z^{-1}}
$$

The full sequence of the above process can be seen in Fig. 1.

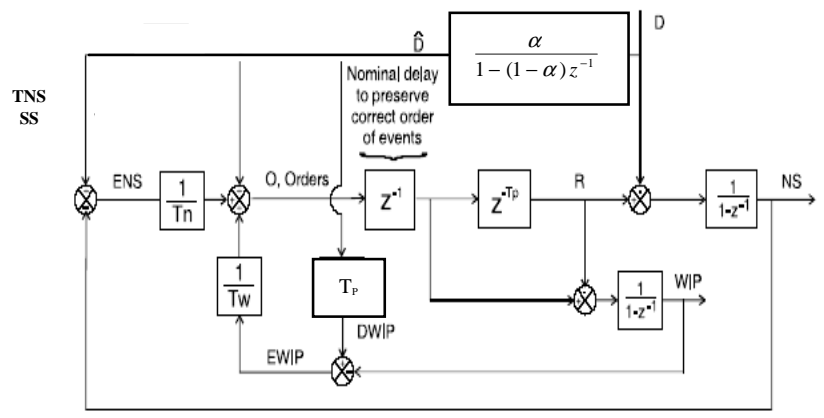

Figure 1: Block diagram for the order up to policy using the fractional replenishment rule.

\section{Stability analysis}

In [4], the above model was analyzed in terms of time response, however a stability analysis for the system was not investigated for different values of the system parameters. Therefore, to examine the system stability, it can be seen from Equation (11) that the system multiple poles depends on $T_{p}$. However, there is a separate pole which is always stable as it achieves the following condition

$$
z=\frac{T_{a}}{\left(1+T_{a}\right)}<1
$$

which will hold for all values of $T_{a}$. The stability of the other poles can be individually investigated using the following theorem. 
Theorem 1: If the replenishment rule transfer function (between the demand and the orders) is represented by Equation (11) and $T_{n} T_{w}>\left|\left(T_{n}-T_{n} T_{w}\right)\right|+\left|\left(T_{w}-T_{n}\right)\right|$ then replenishment rule is stable.

Proof: From Rouche's theorem, if the characteristic polynomial is represented as follows

$$
H(z)=a_{0} z^{n}+a_{1} z^{n-1}+a_{2} z^{n-1}+\cdots a_{n}
$$

with $a_{0}>0$, then for the system poles to lie in the unit circle it is sufficient that

$$
\left|a_{0}\right|>\sum_{i=1}^{n}\left|a_{i}\right|
$$

applying this theorem to Equation (11), thus the replenishment rule is sable if

$$
T_{n} T_{w}>\left|\left(T_{n}-T_{n} T_{w}\right)\right|+\left|\left(T_{w}-T_{n}\right)\right|
$$

which completes the proof. This could hold for a wide range of $T_{n}$ and $T_{w}$ which indicates that the stability of the proposed system could be guaranteed depending on the selection of $T_{n}$ and $T_{w}$. Note that in the following sections, and for simplicity, $T_{p}$ is considered to be 1 .

\section{GENETIC ALGORITHM (GA)}

GA is a general technique for searching a solution space in a manner analogous to the natural selection procedure in biological evolution [10]. Conventional search techniques are often incapable of optimizing non-linear multi-modal functions $[11,12,14]$. GA differs from many traditional optimization algorithms in that the latter usually suffer from myopia for highly complex search spaces [11, 12]. In such cases, a random search method might be required. Gas do not use much knowledge about the problem to be optimized and do not deal directly with the parameters of the problem. They work with codes, which represent parameters. The parameters to be optimized are usually represented in a string form since genetic operators are suitable for this type of representation (binary or integer representation method).

\section{Minimising the bullwhip effect}

The GA is used to select the best parameters which are able to reduce the bullwhip effect and compared with that mentioned in [4]. The following cost function is considered

$$
\begin{aligned}
& J_{B L}=\sum_{k=0}^{M} e^{2}(k) \\
& \text { s.t. }\left\{\begin{array}{l}
T_{W_{\min }} \leq T_{W} \leq T_{W_{\max }} \\
T_{n_{\min }} \leq T_{n} \leq T_{n_{\max }} \\
T_{a_{\min }} \leq T_{a} \leq T_{a_{\max }}
\end{array}\right.
\end{aligned}
$$


where $e(k)$ is the difference between the desired demand and the estimated orders. For simulation purposes the parameters are $T_{a}$, and $T_{w}$ and $T_{n}$ are subjected to the above constraints.

\section{Simulation Example}

Applying the GA to the above cost function (Equation (14)) considering the system in Equation (11), and using the following constraints

$$
\left[\begin{array}{l}
0 \\
0 \\
0
\end{array}\right] \leq \begin{gathered}
T_{W} \\
T_{n} \\
T_{a}
\end{gathered} \leq\left[\begin{array}{l}
100 \\
100 \\
100
\end{array}\right]
$$

the tuning parameters are found to be $\alpha=0.8365, T_{n}=99.9717, T_{w}=6.2533$, which have provided the minimum bullwhip effect (error) over the simulation time. It is clear that the obtained parameters satisfy the developed stability condition in Theorem 1 (see Equation 12) which will be the same. Examining the effect of the determined parameters on the bullwhip effect and comparing them with those suggested in [4] ( $\left.\alpha=0.1111, T_{n}=8, T_{w}=8\right)$. Figure 2 shows the orders with respect to unit step in demand (system step response).

It is clear that the determined parameters (using GA) has almost eliminated the bullwhip effect especially if compared to that obtained when using those mentioned in [4] over the simulation time. However, Figure 3 shows that the net stock required a very long time to recover. This leads to an extra cost due to the time taken for the net stock to recover completely from this step input signal. Consequently, the inventory related costs will be larger. This can be regarded to the proposed cost function that considers only $e(k)$ without paying any attention to the effect of the time taken by the net stock to recover from the demand. This argument is worth trying to show that, in case of using replenishment rule, there is a tough trade off to be made between minimizing inventory holding and shortage costs on the one hand and production switching cost on the other side. This can be achieved by adding a new term that describes the effect of the recovery time to the proposed cost function as follows

$$
\begin{aligned}
& J_{B L}=\left(\sum_{k=0}^{M} e^{2}(k)\right)+T_{\text {recover }} \\
& \text { s.t. }\left\{\begin{array}{l}
T_{W_{\min }} \leq T_{W} \leq T_{W_{\max }} \\
T_{n_{\min }} \leq T_{n} \leq T_{n_{\max }} \\
T_{a_{\min }} \leq T_{a} \leq T_{a_{\max }}
\end{array}\right.
\end{aligned}
$$

where $T_{\text {recover }}$ is the time taken by net stock to recover from the input signal (demand). Again, applying the GA to Equation (17), considering the same constraints in Equation (16). The system tuning parameters are found $\alpha=0.854, T_{n}=6.1707, T_{w}=4.5889$. The system response using the above parameters are shown in Figures $\mathbf{4}$ and $\mathbf{5}$. It can be seen that the bullwhip effect is slightly increased while the recovery time is decreased. 
The new results are still not satisfactory. Therefore, additional consideration should be taken into account and a robust technique such as DDGPC will be introduced to Equation (11) using the lat latter results.

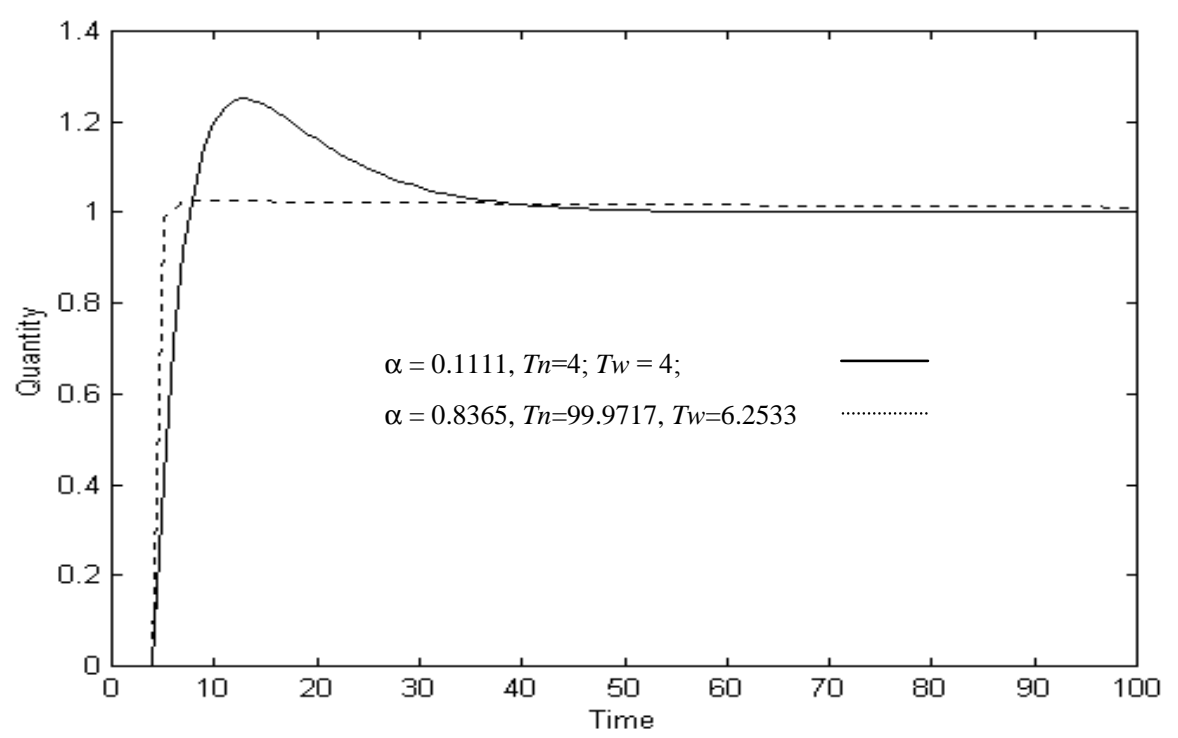

Figure 2: System Order with respect to step demand (GA and Eq. 14).

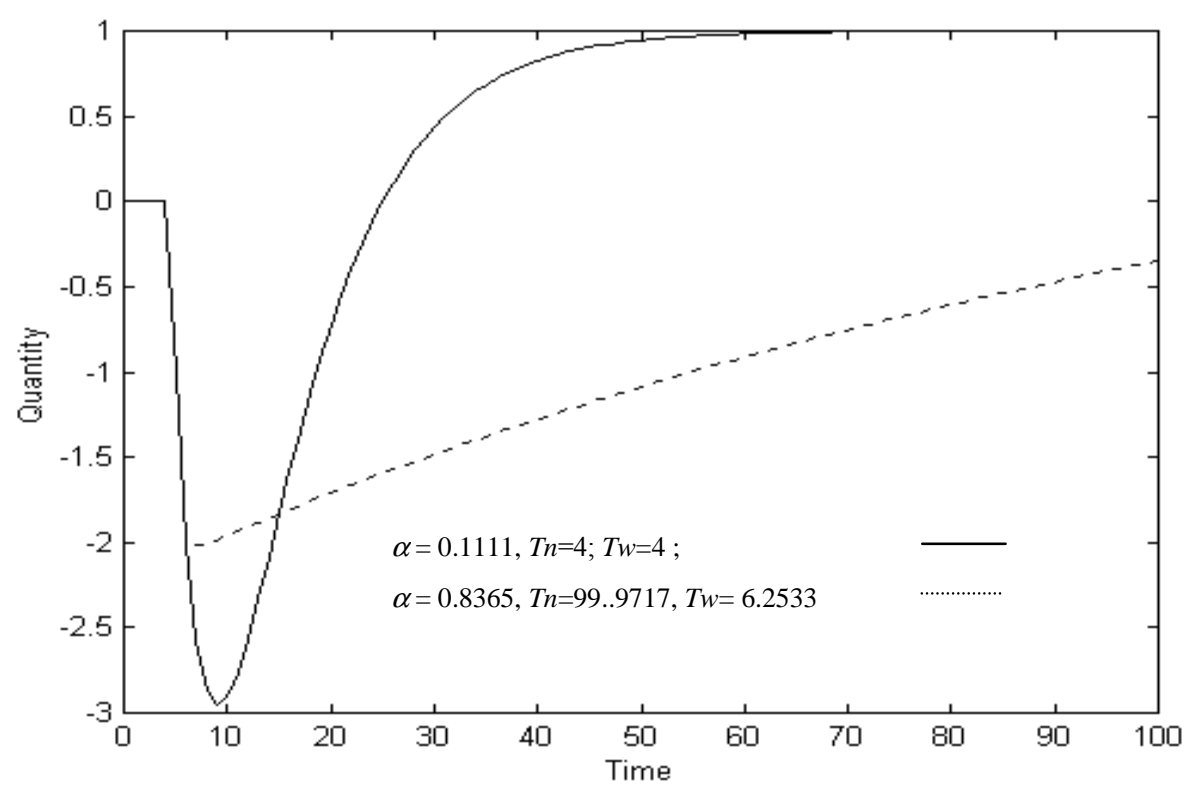

Figure 3: Net stock response (GA and Eq. 14). 


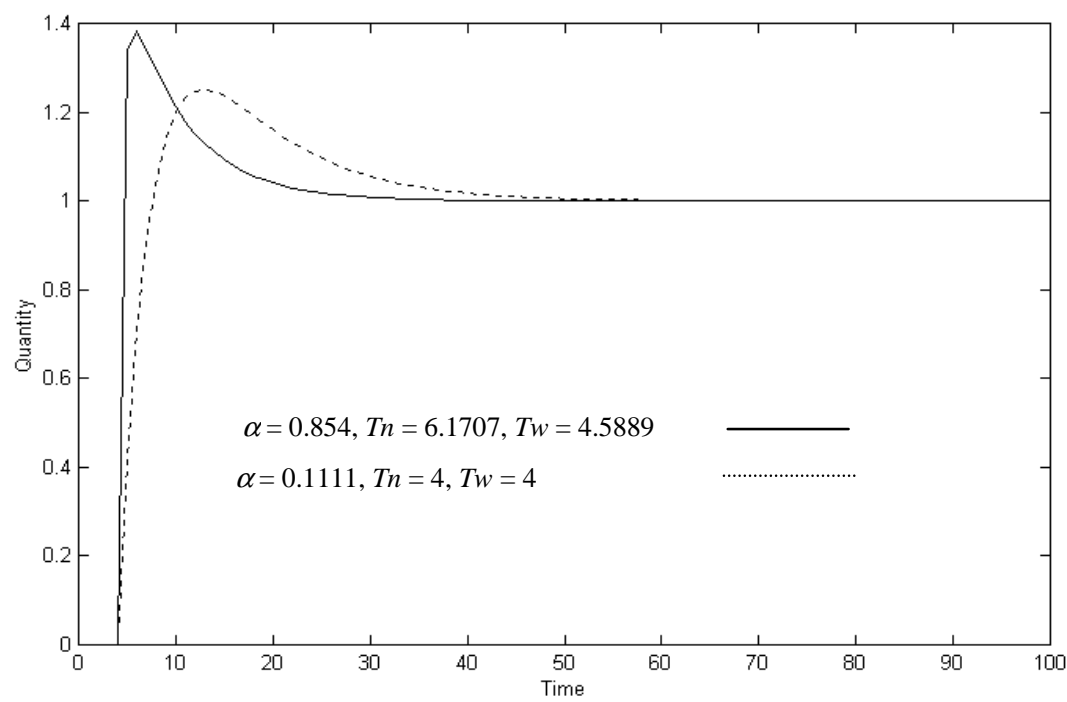

Figure 4: System Order with respect to step demand using GA and Eq.17.

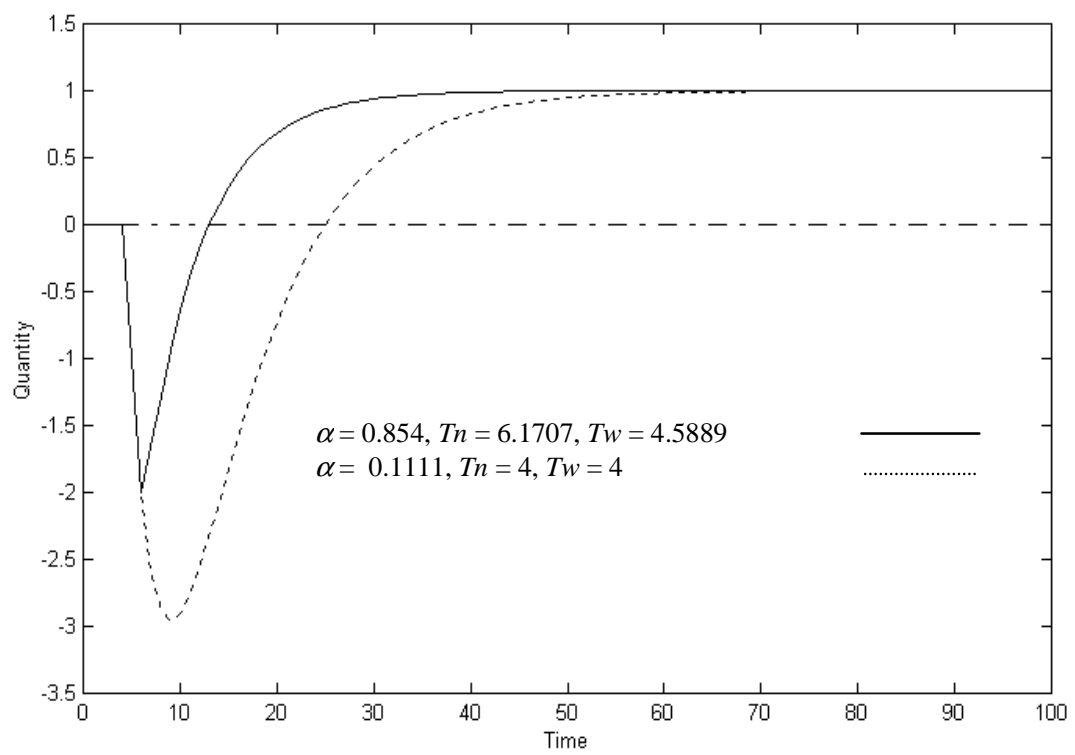

Figure 5: Net stock response using GA and Eq.17.

\section{CONTROLLER DESIGN}

From the above it is clear that using GA has a significant impact on reducing the bullwhip effect when the replenishment rule is used for the ordering process. However, the observed bullwhip effect may vary according to the system uncertainties. Therefore, applying a robust control technique such as DDGPC could lead to a better response. 
The controller will be designed based on the model given in Equation (11) using the above parameters $\alpha=0.854, T_{n}=6.1707, T_{w}=4.5889$ (GA model). The aim of the controller is to produce a suitable control signal (Demand signal) in a fashion that will reduce the observed variance amplification. However, it is useful to design a controller, which provides not only acceptable performance but the stability is also a paramount issue which should be considered. Therefore, this section provides a sufficient condition for stability. This condition will be in terms of controller's gains and system transfer function. It is understandable that these gains are functions of design parameters. Thus, stable systems can be obtained by adjusting these parameters.

Theorem 2: In DDGPC if the closed loop system for the nominal plant (replenishment rule model) $\frac{B}{A}$ can be represented as follow

$$
y(t)=\frac{B\left(z^{-1}\right)}{A\left(z^{-1}\right)}\left[Q\left(z^{-1}\right) y(t)+S\left(z^{-1}\right) y(t)+r(z) \omega(t)\right]
$$

where

$$
\begin{aligned}
& y(t)=G\left(z^{-1}\right) u(t) \\
& K_{0}\left(z^{-1}\right)=k_{0,0} z^{-1}+k_{0,1} z^{-2}+\cdots+k_{0, n a} z^{-n a} \\
& K_{1}\left(z^{-1}\right)=k_{1,0} z^{-1}+k_{1,1} z^{-2}+\cdots+k_{1, n a} z^{-(n b+1)} \\
& R(z)=r_{1} z+r_{2} z^{2}+\cdots r_{N_{2}} z^{N_{2}}
\end{aligned}
$$

For stability, it is sufficient that

$$
|Q-S|<\frac{A}{B} \quad \forall 0<\omega<\frac{\pi}{T}
$$

\section{Proof}

The characteristic equation closed loop system can be written as:

$$
F=1-\frac{B}{A} Q-S \frac{B}{A}
$$

It is worth mentioning that the shift operator $z^{-1}$ has been dropped for clarity. For the closed stability, the characteristic equation must satisfy the following:

$$
\left|1-\frac{B}{A} Q-\frac{B}{A} S\right| \neq 0 \quad \forall|z| \geq 1
$$

which holds if

$$
\left|1-\frac{B}{A} Q-\frac{B}{A} S\right|>0
$$

which will hold if

$$
|Q-S|<\left|\frac{A}{B}\right|
$$


which provides sufficient condition for stable closed loop system and completes the proof.

\section{Robustness to system uncertainties}

The robustness of closed loop system is a vital issue in the design of control systems. This section investigates the robustness of the proposed controller when applied to the proposed replenishment rule model. The most uncertain parameter in the proposed model is $T_{\mathrm{p}}$. It is clear from above that different values of $T_{\mathrm{p}}$ leads to different number of poles. To investigate this uncertainty, it is assumed that the real replenishment rule model $\frac{B_{r}\left(z^{-1}\right)}{A_{r}\left(z^{-1}\right)}$ is related to the nominal model by

$$
\frac{B_{r}\left(z^{-1}\right)}{A_{r}\left(z^{-1}\right)}=\frac{k B\left(z^{-1}\right)}{A^{+}\left(z^{-1}\right) A\left(z^{-1}\right)}
$$

where $A^{+}\left(z^{-1}\right)$ contains the unmodeled poles due to uncertain $T_{\mathrm{p}}$ and $k$ is added in the numerator so there are no discrepancies between the static gain of the real model and the nominal one. The following Lemma studies the influence of unmodeled poles on the system stability.

\section{Lemma 1}

In DDGPC the control signal is found to be

$$
u(t)=\left[Q\left(z^{-1}\right) y(t)+S\left(z^{-1}\right) y(t)+R(z) \omega(t)\right]
$$

and the replenishment-rule real model can be represented as in Equation (24), where $A^{+}$contains unmodeled poles due to uncertain $T_{p}$. For stability it is sufficient that

$$
\frac{|B Q-B S|}{|A|}<|A|^{+} \quad \forall 0<\omega<\frac{\pi}{T}
$$

\section{Proof}

The characteristic equation closed loop system can be written as:

$$
F=1-\frac{B}{A^{+} A} Q-\frac{B}{A^{+} A} S
$$

It is worth mentioning that the shift operator $z^{-1}$ has been dropped for clarity and the gain $k$ in Equation (24) has been absorbed in the polynomial $B$. For the closed stability, the characteristic equation must satisfy the following:

$$
\left|1-\frac{B}{A^{+} A} Q-\frac{B}{A^{+} A} S\right| \neq 0 \quad \forall|z| \geq 1
$$

which holds if 


$$
\left|1-\frac{B}{A^{+} A} Q-\frac{B}{A^{+} A} S\right|>0
$$

which will hold if

$$
\frac{|B Q-B S|}{|A|}<\left|A^{+}\right|
$$

which provides sufficient condition for stable closed loop system and completes the proof.

\section{SIMULATION RESULTS}

Considering the above, the DDGPC controller will be derived here for the parameters $N_{1}=1, N_{2}=3, N_{u}=3, \delta=1$ and $\lambda=6$. The designed controller will be applied to the replenishment rule [4] and a comparison will be conducted between the controlled model and the uncontrolled ones.

\section{Time response analysis}

Figure 6 shows the step responses for replenishment rule in three cases, namely, the controlled GA model, GA model and the original model (Dejonkheere et al [4]).

It should be mentioned that the simulation of the previous section is repeated here for clarification. It is clear that the controlled system provides the least overshoot for the generated orders, while the uncontrolled one has a noticeable overshoot. This indicates the ability of the controller to minimize the bullwhip effect. On the other hand, Figure 7 shows that the controlled system when compared with the uncontrolled GA model takes slightly longer to recover from this step input signal. However, it has a significant improvement when compared with the Dejonkheere model. This indicates that the controlled GA system satisfy the best trade off between minimizing inventory holding and shortage costs and the production switching costs.

To support the above results, frequency analysis for the above model will be introduced in the next section to show the efficiency of each model in providing a global bullwhip effect reduction over the whole frequency range.

\section{Analysis using frequency response}

The analysis of the bullwhip can be done using the frequency response $[4,5]$. It is shown that the conventional way (ratio of the variance of the orders being generated to the variance of the demand input) to measure the bullwhip is exactly the frequency response of the system transfer function (output/input). Therefore, the bullwhip effect can be detected from the frequency response for different demand frequencies. However, as the real demands are rarely pure sinusoidal, it is preferred to determine the area under squared frequency response curve as another metric for the bullwhip effect.

Using the above, it can be seen from Figure 8, that the controlled system would provide less bullwhip for most range of frequencies. 


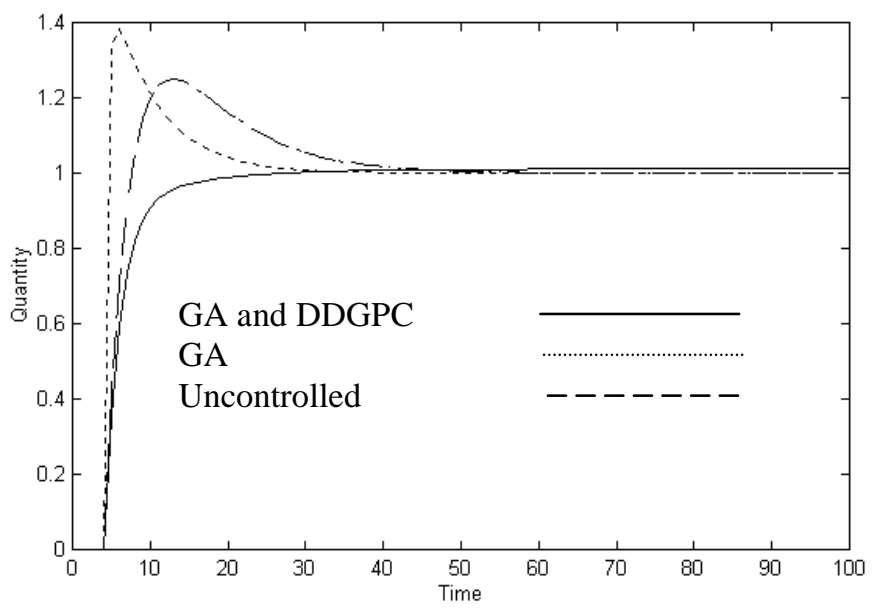

Figure 6: System Order with respect to step demand.

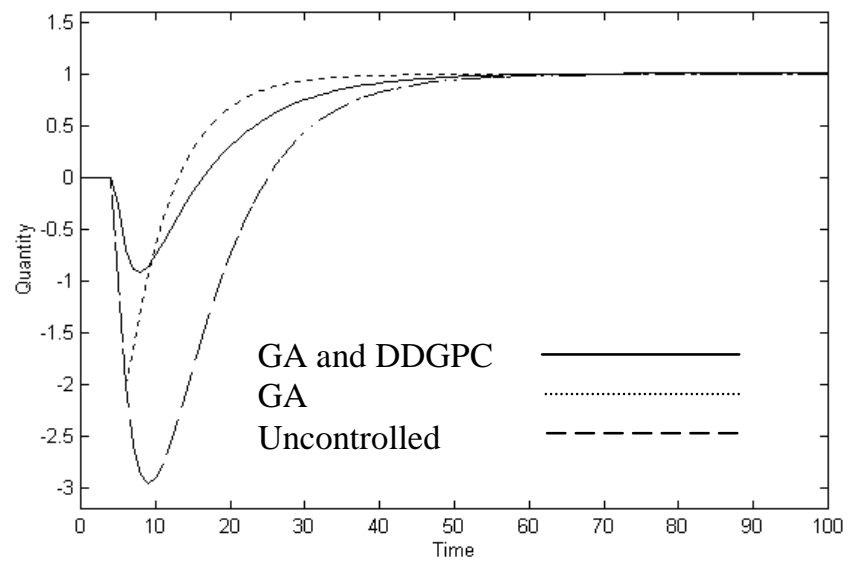

Figure 7: Net stock.

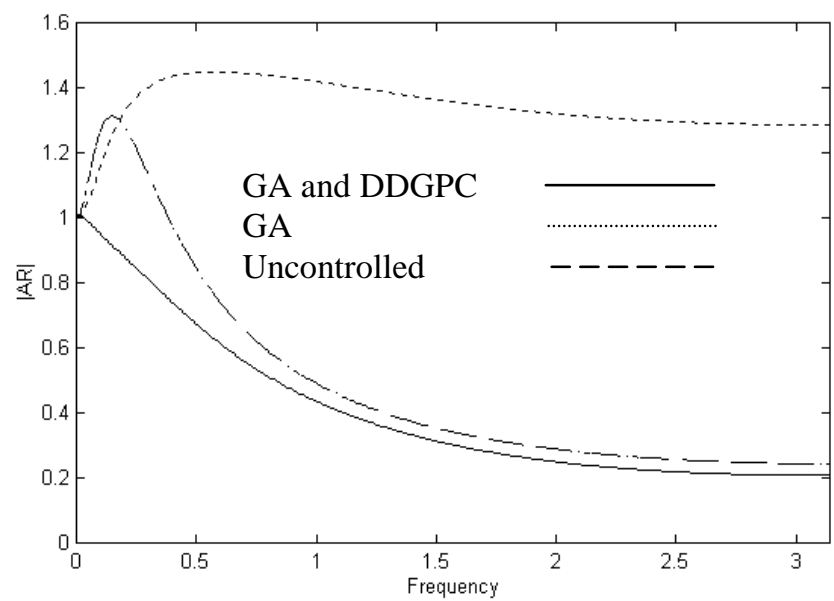

Figure 8: Frequency response. 
This can be supported by calculating the area under the curve for all cases considering. In order to achieve this task a numerical approach for the integration (trapezoidal summation with 0.01 step) for frequency range from 0 to $\pi$ is used. This shows that, the areas under the curve are 4.2044, 1.5337 and 1.2644 for the GA model, Dejonkheere model, and the controlled GA respectively. This indicates that the DDGPC controller is able to reduce the bullwhip effect which sustains the results of the time response.

\section{CONCLUSION}

In this paper, the GA has been introduced to the replenishment rule. The GA has successfully reduced the unavoidable result in a bullwhip effect when demand has to be forecasted. However, the GA has not managed to maintain low inventory cost. Hence, the DDGPC is proposed to overcome this drawback. The DDGPC has shown a significant ability in solving conventional supply chains problems without adding complexity to the model. In addition, analyses of the system stability and robustness have been also investigated to avoid any drawbacks that may appear due to system uncertainties.

\section{REFERENCES}

[1] Forrester,J., "Industrial Dynamics, A major breakthrough for decision makers", Harvard Business Review, July-August, pp. 67-96, 1958.

[2] Forrester,J., "Industrial Dynamics", MIT Press, Cambridge, MA, 1961.

[3] Lee, H. L., Padmanabhan, V., and Whang, S., "The bullwhip effect in supply chains", Sloan Management review, pp. 93-102, 1997.

[4] Dejonckheere, J., Disney, S.M., Lambrecht, M.R., and Towill, D.R., "Measuring and avoiding the bullwhip effect: A control theoretic approach", European Journal of operational research, 147, pp. 567-590, 2003.

[5] Dejonckheere, J., Disney, S.M., Lambrecht, M.R., and Towill, D.R., "The impact of information enrichment on the bullwhip effect in supply chains: A control engineering perspective", European Journal of operational research, 153, pp. 727750, 2004.

[6] Gomma, H. W. and Owens, D. H., "Delayed Generalized Predictive Control", IEE International Conference on Control'98, No. 455, pp. 793-797, 1998.

[7] Yu, H., Gomma, H. W., Cang S., Chen, W., and Yusuf, Y., "Reducing Bullwhip Effect Using Delayed Generalized Predictive Control", IEEE International Conference on Systems, Man and Cybernetics, October 10-13 2004, The Hague, The Netherlands, pp. 1510-1515, 2004.

[8] Sterman, J., "Business dynamics, system thinking and modeling for a complex world", Irwin McGraw-Hill, New York pp.982, 2000.

[9] Gomma, H.W., and Yu, H., "Derivative Derived Generalized Predictive Control (DDGPC) a technique for improving performance", IEEE CCA 2005, Canada, pp.-1152-1157, 2005.

[10] Holland, J. H., "Adaptation in Natural and Artificial Systems", Ann Arbor, MI: Univ. MichiGAn Press, 1975. 
[11] Miller, J. A., Potter, W. D., and Gandham, R. V., "An evaluation of local improvement operators for genetic algorithms," IEEE Trans. Syst., Man, Cybern., vol. SMC-23, no.5, , pp. 1340-1350, 1993.

[12] Salomon., R., "Evolutionary algorithms and gradient search: similarities and differences," IEEE Trans. Evolutionary Computation, Vol.2, No.2, pp. 4555,1998 .

[13] Towill, D.R., "Dynamic analysis of an inventory and order based production control system," International Journal of Production Research, 20, pp. 369-383, 1982.

[14] Carrillo-Ureta, G. E. $\square$, Roberts , P.D. $\square$ and Becerra , V. M., "Genetic Algorithms for Optimal Control of Beer Fermentation", IEEE CCA, Mexico, pp.391-396, 2001.

\section{استخدام نظرية الجينات الوراثية والتحكم التفاضلي التوقعي العام في إنقاص تبعات ضربة السوط والن النئ}

في سلاسل الإنتاج لاقى مفهوم ضربة السوط وهو التغيرات الضخمة في أوامر الإنتاج

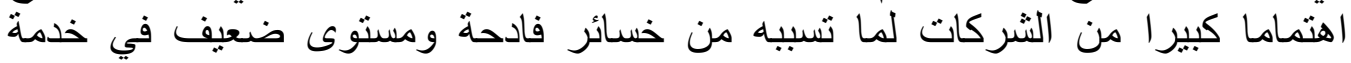

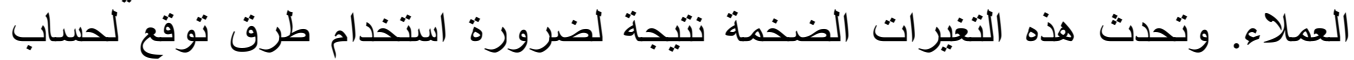

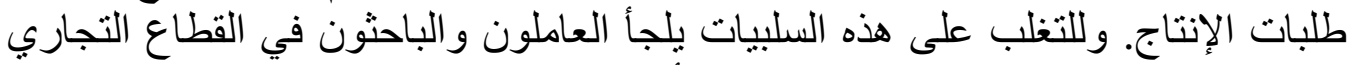

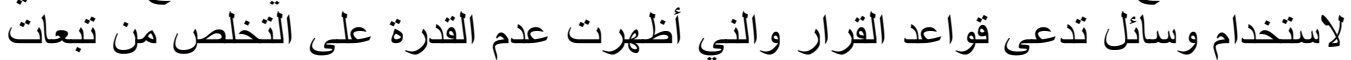

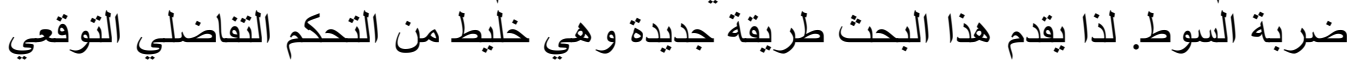

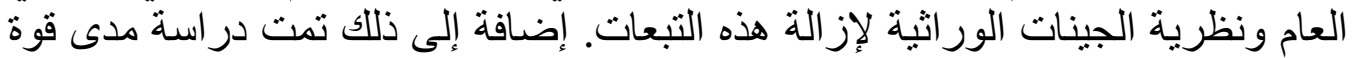

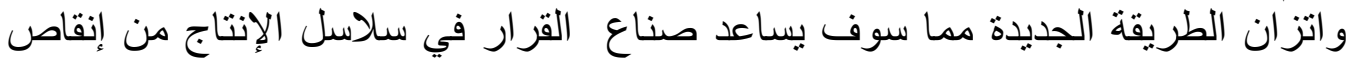
العو اقب الوخيمة الناتجة عن ما يسمى ضرئه فربة السوط. 